\title{
Airwaves
}

No sense of self-preservation?

The editors were surprised to learn that over $40 \%$ of patients with COPD have positive anti-nuclear antibodies. This feature was not associated with smoking status, gender or severity of airflow limitation but did occur more commonly in patients with a low BMI. Whether the presence of positive anti-nuclear antibodies reflects a non-specific effect of tissue damage or an important and modifiable pathogenic pathway is not known. Selfperpetuating autoimmune inflammation could explain a number of features of established COPD including the continuation of airway inflammation and progressive airflow obstruction despite smoking cessation, and the presence of activated oligoclonal CD4+ lymphocytes within the airways. COPD and autoimmunity does seem to be an area which is gathering steam-or will it prove to be mere hot air? More work is needed. See page 101

\section{Losing the lottery}

Readers will be aware that occupational asthma can lead to long-term disability, unemployment and loss of income but will be surprised by the size of the financial costs. Ayres et al estimate that one case of occupational asthma costs the individual the equivalent of winning the lottery ( $£ 13$ million) over the course of his/her lifetime. Society contributes a similar amount but employers bear only a small fraction of the total cost $(3 \%)$. How appropriate is this? In an accompanying editorial, Burge argues that this disproportionately small financial penalty provides little incentive for employers to control the cause of occupational asthma. The priority has to remain the primary prevention of new cases and we need to devise better incentives for employers to participate in this process. We should also look again at the ways we compensate the damaged worker. This is an important and difficult area; we will be returning to it in 2011. See pages 92 and 128

\section{The curious incident of the dog in the night-time}

Sherlock Holmes devotees will well remember that the dog doing nothing in the night-time was exceedingly curious. For obvious reasons, little is known about the airway wall pathology in very young children who wheeze. A collaboration between Helsinki and the Brompton previously reported on severely symptomatic infants median age 12 months, referred for investigation including bronchoscopy. As with the surprisingly nonbarking dog, there was no evidence of airway wall inflammation or remodelling, demonstrating that whatever was driving symptoms at this early stage, it was very different from the eosinophilic inflammation and structural changes seen in established asthma. The group now reports on follow-up to age 3 years. The salient points are that 2 years on from the original study, there was still a high burden of symptoms and medication use in this group; and that low lung function in infancy, increased reticular basement membrane thickness, and mucosal mast cell numbers but not eosinophilia were associated with the purchase of ICS in the ensuing two years. So is the mast cell at the route of persistence of symptoms? Do we now need to do a randomised controlled trial with cromoglycate in high risk infants? The safety profile of cromoglycate is appealing, especially as the study would probably have to start early in life. Or will this be another flatter to deceive cromoglycate study? See page 157

\section{Can't they count? Sweet seventeen}

One, two, nine, seventeen: the connection being populations of $\mathrm{T}$-helper cells and immunologists with large brains who have numeral apraxia-don't they know that three then four comes after two?
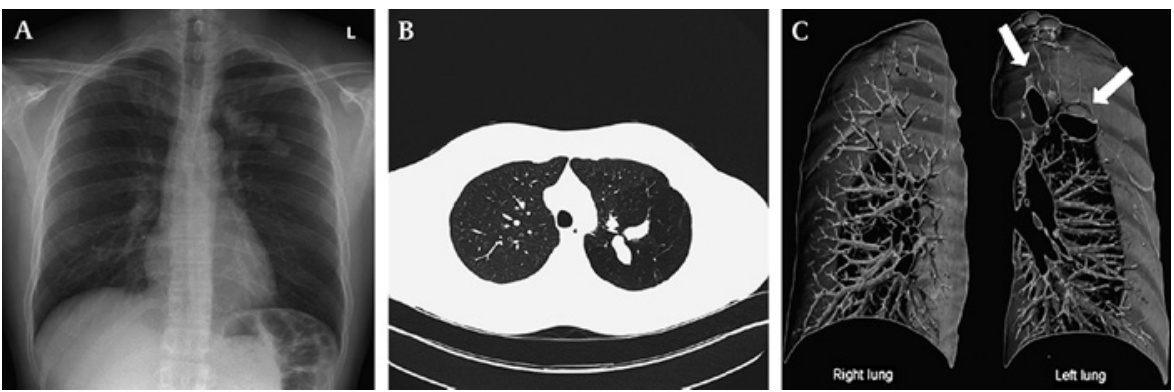

The $T_{H} 17$ population develps in response to IL-2 and IL-1 $\beta$ and expresses among other receptors, CCR6, the only known receptor for CCL20. These cells have been implicated in a number of chronic inflammatory conditions, in particular those associated with $\mathrm{T}_{\mathrm{H}} 1$ overexpression. In this issue, Facco et al showed that $\mathrm{T}_{\mathrm{H}} 17$ cells are found surrounding the central core of the sarcoid granuloma. They are present in the lungs of relapsed patients, absent in those who have remitted. They also suggest that CCL20 may be an important driver of this response. The data raise intriguing possibilities about the pathophysiology of sarcoidosis, but a word of caution; just because $T_{H} 17$ cells are present does not mean they are the bad guys. $\mathrm{T}_{\mathrm{H}} 17$ cells may have important roles in host defence against bacterial infection, for example. Facco et al suggest we may in the future be blocking IL-17 effects, and indeed this may be so. However, their findings could mean that $T_{H} 17$ cells are part of a beneficial host response to control sarcoid, or even eliminate the putative infecting agent causing the disease, and the future might actually be amplifying the $T_{H} 17$ response. As always with the best studies, intriguing challenges are thrown up. See page 144

\section{And finally: two (or more) fingers} up to all our readers!

HIV lung complications comprise a huge spectrum of infective, neoplastic and iatrogenic disease. The images below are from a 31 year old HIV positive man who presents with a dry cough. What do they show, and what would you do? Answers before turning to Page 182 